\title{
Adjustment and the Near-Death Experience: A Conceptual and Therapeutic Model
}

\author{
Bette G. Furn, Ph.D. \\ Counseling Services, University of Hartford
}

\begin{abstract}
Most mental health practitioners, and counseling psychologists in particular, possess skills for helping near-death experiencers. What is needed is a conceptual framework that is both familiar to practitioners and highly relevant to that client group. Cross-cultural counseling in general, and the consideration of world views more specifically, are suggested. Using that framework, the world view assimilated during the near-death experience (NDE) is viewed as being in contrast to that of the "old self," significant others, and the majority culture. The difficulties reported by NDErs are considered analogous to those associated with culture shock. The world views of the practitioner, NDEr, and relevant others should be taken into account in the formulation of psychoeducational and therapeutic interventions.
\end{abstract}

The specific question being considered in this essay is: in what ways can counseling psychologists aid near-death experiencers (NDErs) in adjusting to the experience and its aftereffects? While the focus is on the particular skills and orientations of counseling psychologists, it is hoped that the model to be presented will prove useful to a wide variety of practitioners working in medical and other health-care settings. Each aspect of the question will be considered separately, with all relevant terms defined. The final section will suggest a paradigm from which to view the particular adjustment problems of the NDEr, and from which to draw appropriate intervention strategies.

A major premise of this paper is that most mental health practitioners, and counseling psychologists in particular, should already posses the

Requests for reprints should be sent to Dr. Furn at the Counseling Services, University of Hartford, West Hartford CT 06117. 
requisite skills for aiding this population; what is needed is a conceptual framework that is, or should be, both familiar to the practitioner and highly relevant to the client group. Such a paradigm can be provided by the literature on cross-cultural counseling.

\section{Counseling Psychology as an Applied Discipline}

The historical roots of counseling psychology can be traced, in part, through the mental hygiene movement of the early twentieth century. While this, in and of itself, is not a particularly exclusive distinction, it has been counseling, more than other branches of psychology, that derived its heritage from nonmedical and nonpsychoanalytic traditions (Whiteley, 1984). Initially through the vocational guidance movement, and later via the client-centered approach of Carl Rogers, counseling psychology developed as a discipline concerned with life-stage issues of subjective import to its client population. Historically, its focus has been developmental, and remediation has been approached largely through a psychoeducational model. The American Psychological Association (1981) refers to services by counseling psychologists as those for "facilitating effective functioning during the life span developmental process ... with a significant emphasis on positive aspects of growth and adjustment and with a developmental orientation" (p. 654).

With some significant omissions, the above description captures the essential spirit of counseling psychology as an applied discipline. While it may seem that what has been presented is a thinly veiled example of professional chauvinism, it is important to recall, first, that many of the specifics of orientation and technique are not unique to counseling psychologists, and, second, that the conceptual framework as it has been outlined thus far allows for an easy inclusion of the near-death experiencer as an appropriate client, if one is willing to consider an encounter with death as a life stage.

For reasons that should become clearer in the following sections, it is particularly important that interventions designed for the NDEr be generated from a paradigm primarily concerned with adjustment issues of people in general, as opposed to those primarily concerned with atypical response patterns. A framework derived from nonmedical and nonpsychoanalytic traditions should not only be more appropriate to this client group, but could also free the practitioner from many of the inherent biases of orientations more accustomed to the diagnosis of disease and psychological pathology.

The historical legacy of counseling psychology has called for the pro- 
vision of psychoeducational services for a broad range of developmental and adjustment issues. The mandate for the future is to anticipate new client populations and to address their special needs (Whiteley, 1984). The near-death experiencer represents such a client group.

\section{The Near-Death Experience}

Interest in the near-death experience (NDE), as such, is a relatively new phenomenon, beginning in this country some fifteen years ago with the work of Raymond Moody $(1975,1977)$ and Elisabeth Kübler-Ross. Since their pioneering efforts, a number of investigators (Gallup, 1982; Greyson, 1982; Greyson \& Flynn, 1984; Ring, 1980, 1984; Sabom, 1981) have significantly elaborated upon the initial descriptive work, employing more scientifically rigorous methodology. Both types of inquiry have yielded highly consistent findings.

In general, the NDE may be thought of as a process that occurs for some individuals as a result of coming close to death, or, perhaps, of being clinically "dead" for a period of time. Kenneth Ring (1980) related a thorough composite description of what might be considered a "typical" NDE, based on more than one hundred interviews with near-death survivors. While the reader is encouraged to consult that source, a brief outline of the experience is appropriate here.

Commonly, the early stage of the experience is accompanied by feelings of peace and quiet, perhaps also involving the viewing of one's own body and the surrounding area from a vantage point somewhere above. The individual may then hear a buzzing or other similar sound, and be aware of moving or being drawn through a dark tunnel or some other "passageway." In the deeper stages of the experience, the individual may encounter an exceptionally brilliant light, which often is perceived as encompassing, or actually being, some sort of a presence. The "light" may afford the experiencer the opportunity to review his or her life, as if in a replay that is vividly clear despite an instantaneous quality. The review allows for a critical attitude toward the details of one's own life, while, nonetheless, occurring in an atmosphere of total love and acceptance. At that point, in general, the experiencer becomes aware that a decision will be made as to whether he or she will stay or return. In some instances the experiencer chooses, while in others there is no option. The "return" to the physical body is abrupt, usually without awareness of how one was returned.

What happens later will be covered in greater depth in the section on aftereffects; at this point, however, it is appropriate to say that the 
experiencer, if prone to discuss the matter at all, finds great difficulty in adequately communicating the nature and quality of the experience. There is a profound subjective sense of its ineffability, but with no doubt as to its reality.

The above description notwithstanding, the reader is urged to recall that all human experience involves variability; the near-death experience is no exception. Not all NDEs follow the pattern precisely, and perhaps more significantly to the mental health practitioner, not all NDErs respond to it in a uniform fashion.

It is estimated that somewhere between $35 \%$ and $40 \%$ of people who have been close to death could report NDEs (Ring, 1984). Using George Gallup's (1982) estimates, that could involve roughly 8 million adult Americans. Given the ever-increasing sophistication of medical technology, it is reasonable to assume that the number has increased, and is likely to continue to do so. It is also quite likely that a wide variety of individuals, who may come close to death through any number of circumstances, could potentially undergo the experience. The research literature thus far has shown that none of the commonly considered demographic variables (age, sex, race, socioeconomic status, educational level, marital status, religious affiliation, geographic variables) are significantly related to the occurrence or content of the NDE. Neither does the manner in which one comes close to death seem to affect the experience. Little research is available, however, on populations significantly different from those of the Western world. That not only leaves a question as to the universality of the experience, but also, for the practitioner, warrants a caveat as to what assumptions one might make when working with someone from a non-Western heritage.

\section{The Aftereffects}

As one might expect, being subject to such a profound and "unusual" happening may present problems. Although much of the research literature to date has catalogued, fairly extensively, what the aftereffects are, there is some disagreement as to whether the list is complete, and to what extent it is biased in emphasizing the positive. Any "transformation" that occurs in the life of the NDEr may be a process involving years.

While it may seem premature to express such strong reservations prior to outlining just what the aftereffects are, it is crucial, $I$ believe, in aiding this population, that the interventionist be cautioned against colluding in a kind of "pedestal effect." For reasons that should become clearer later in this section, that may be difficult to do. 
The research literature previously cited in the text will serve as the primary sources for summarizing the aftereffects of the NDE. In order to present the material in a fashion more meaningful to the practitioner, it will be organized according to the following categories: personal, interpersonal, and cultural aftereffects. While some of these might reasonably be included in more than one category, the distinction should prove pragmatically useful.

\section{Personal Aftereffects}

Most sources consulted agree that the NDEr is likely to feel a sense of loss at having to relinquish the incredible beauty and peace encountered in the "other world." He or she is relegated to an existence that now seems terribly mundane by comparison. Sorrow, anguish, and the inability, for many, to be able to successfully communicate to others the profundity of what has occurred, often may signal the beginning of a major, clinical depression. (It is important to note that the depression, as experienced by the NDEr, will differ in some significant ways from the more typical case, in terms of diagnostic criteria [American Psychiatric Association, 1980]; for example, feelings of worthlessness, excessive guilt, and suicide ideation are highly unlikely. The affective pain, however, is as intense.)

A corollary problem is presented by virtue of not being able to communicate the nature of the experience. If the NDEr does make an attempt to share what has just transpired, it is likely that the response will be one of disbelief, of greater or lesser intensity. This not only serves to further isolate the NDEr emotionally, contributing to the depression, but also fuels the fear of being thought crazy. This is a significant and persistent risk. In addition, some NDErs report general difficulty in coping with the rudiments of language (Atwater, 1983), as opposed to the instantaneous and total comprehension that was present during the NDE. Taken all together, these "symptoms," which follow from the context of the individual's close encounter with death, make him or her seem a likely candidate for a psychiatric referral. Depending upon the frame of reference of the consulting professional, if indeed one is contacted, that could spell disaster or relief for the experiencer.

The aftereffects described thus far generally begin to surface in the period immediately following the NDE; others become apparent over varying lengths of time. Not the least of these is a changed attitude toward death. People who have reported a near-death experience show a marked and lasting decline in their fear of death. It would not be over- 
stating the case to say that, for most, all fear of death is gone. This is not to say that the process of dying is viewed totally without dread, but that actually being dead evokes no fear. Concomitantly, there is a marked increase in a belief in life after death, which may be more rightly described as a certainty that there is a life after death.

As one might expect, the NDEr is also likely to undergo religious or philosophical changes. These may include considering oneself as more spiritual than religious, with a reported decreased emphasis on formal aspects of religion. An increased awareness of the presence of God and a tendency toward emphasizing the universals of religion are also cited. For many, that translates behaviorally into a more serene or peaceful demeanor, less interest in material things, a greater appreciation of and need for solitude, and a focus on the values of love, compassion, and giving.

Probably much more difficult for most practitioners to accept are the data reporting heightened psychic abilities, including clairvoyance, telepathy, precognition, synchronicity, and the "witnessing" of future personal events. More rarely, and certainly more disturbingly, NDErs report visions of our planetary future (Ring, 1984). Whether or not one accepts the credibility of these reports, it is fairly easy to see that the NDEr will be dealing with substantial skepticism.

Over what seem to be fairly extended periods of time, the NDEr is also apt to feel a stronger sense of self-esteem and assertiveness. For some, it seems to derive from their direct experience with "the light" and its ambience of total love and acceptance; for others, it may come from the life review and a fresh perspective on psychological encumbrances.

Although the literature to date tends to focus on the more intriguing revelatory and growth-inducing aspects of the NDE, it should be apparent to the practitioner that much of what has been described also falls within the realm of profound psychological distress. Such clinical terms as depression, anxiety, and adjustment reaction could all be appropriately applied, while a host of other more pathological descriptors would probably come to mind. On a philosophical, as well as a deeply personal level, the NDEr has undergone an existential crisis of the first order. A difficulty in adjustment, often enduring for years, is to be expected.

\section{Interpersonal Aftereffects}

Commonly cited, although not thoroughly explored, is the strain upon the primary relationships of the NDEr. Bouts of crying, seemingly in- 
appropriate anger, withdrawal, and other behaviors deemed atypical of the NDEr may surface shortly after the experience, with longer-term value and life-style changes generally evolving more slowly. All of these may be problematic not only for the NDEr but also for family and friends, work colleagues, superiors, and anyone else regularly involved with the experiencer. Reconsider the personal aftereffects outlined above, and recall that the NDE is not significantly correlated with any of the common demographic variables. What that means is that any normal, average individual, having had a close encounter with death, may suddenly sound like, act like, and think like an entirely different, and probably unfathomable person. Unfortunately, that seems to be the rule, rather than the exception.

From the perspective of the NDEr, he or she has been privy to an extraordinary "realm" of experience that has enlightened him or her to a whole new conceptualization of self and others, to what is important and what is not, and so on. Probably in part because of the NDE's ineffability, the experiencer also may feel a sense of exclusivity; even should one try to explain the new frame of reference, no words are adequate, and no one who has not experienced the state could ever really understand. That kind of attitudinal set translates into significant interpersonal behavior problems.

In varying degrees of intensity across the entire post-NDE period, the NDEr is quite likely to have great difficulty in integrating his or her attitudinal and behavior changes with expectancies previously met by the "old self." What used to be routine role functioning, may seem superfluous or insignificant in light of new values and priorities.

From the perspective of significant others, all of that may be perceived as tantamount to interpersonal breach of contract. Imagine for a moment a typical middle-class family whose primary wage earner no longer particularly values the American work ethic, with its emphasis on capitalism and economic growth; who no longer considers community standing as worthy of being bothered about; and who has moved material accoutrements to the bottom of the priority list. Shock, anger, and other similar reactions are probable toward such a unilateral need to alter the familiar, in addition to ambivalence and perhaps guilt at feeling such things toward someone who has nearly died.

Almost paradoxically, the interpersonal strain is rooted in the NDEr's conviction that love and unconditional acceptance of others are paramount values; their translation into the minutest details of everyday life becomes an imperative. The corollary life-styles changes required are perceived as enormous and unreasonable to those more firmly rooted in the trappings of this world. 


\section{Cultural Aftereffects}

In the broadest sense, much of the above reflects a major shift in the frame of reference of the NDEr from the pre-NDE value system to the orientation experienced during the process of the NDE. To the extent that a widely shared value system is synonymous with "culture," it may be said that NDErs have philosophically and behaviorally adopted a new culture. Cultural aftereffects, then, refer not just to transactions between the experiencer and society, but also to a significant shift in his or her world view. The NDEr's conception of self, of others, of nature, of the nature of life, and of time may be significantly altered during a generally extended period following the NDE.

The literature describes what might be considered a more spiritual orientation to life; that is, the NDEr, particularly if he or she has had a deep experience, tends to express a heightened appreciation of or reverence for life, and stresses love as being of the utmost importance. Frequently it also involves a deeply felt need to determine the reason for one's being here and to accomplish whatever one's purpose is. The NDE and its evolving ramifications become central to the way in which the NDEr perceives and relates to the world.

Again, it is important for the practitioner to recall that none of the aftereffects described necessarily pertain to any given individual who has had a close encounter with death. The information presented here is a compilation of data that tends to distinguish NDErs as a group; variation within the group may be considerable. Given the unusual and intriguing nature of the phenomenon, it may be extremely tempting to make unwarranted assumptions about the NDE client. The task of the interventionist should be to educate the experiencer and significant others to what are, or may be, the issues with which they have to cope, and to provide a framework through which to facilitate adjustment.

\section{What Constitutes Adjustment?}

It often seems that an understanding of what "adjustment" is comes most easily by determining what it is not. In a sense, it is conspicuous by its absence. Nonetheless, the term clearly implies an interface with some "other," generally understood to be at the system level. To varying degrees, then, it reflects the congruence of person-environment fit.

As a construct defining an aspect of psychological health, it generally refers to a successful balancing of personal needs, interpersonal obligations, and cultural ethics. Differing formal definitions seem to focus var- 
iously, and subtly, on one or another of the three components. Robert Goldenson (1984) defined adjustment, in part, as "the modification of attitudes or behavior to meet the demands of life effectively, such as carrying on constructive interpersonal relations, dealing with stressful or problematic situations, handling responsibilities, or fulfilling personal needs and aims ..." (p. 16). The focus of that definition is on the cultural level, here referred to as "the demands of life." Benjamin Wolman (1973) shifted the emphasis slightly to the personal level, and defined adjustment, in part, as "an harmonious relation with the environment involving the ability to satisfy most of one's needs and meet most of the demands, both physical and social, that are put upon one ..." (p. 9). A somewhat more equivocal and probably more realistic definition was provided by Hans Eysenck (1972):

1. A state in which the needs of the individual on the one hand and the claims of the environment on the other are fully satisfied. Harmony between the individual and the objective or social environment. 2 . The process by which this harmonious relationship can be attained. The state is of course expressible only in theoretical terms, since in practice no more than a relative adjustment is reached in the sense of optimal satisfaction of individual needs and untroubled relation to the environment. ... (p. 25)

The relativistic and nonjudgmental posture reflected in that definition should appeal to the practitioner interested in devising intervention strategies within a cross-cultural framework. It presupposes no absolute values and acknowledges that adjustment is as much an ongoing process as it is an end state.

As a construct defining psychological disorder, or "maladjustment," it refers to an incongruence in person-environment fit, generally recognized as being of significant proportions and often as being of a temporary nature. The Diagnostic and Statistical Manual of Mental Disorders (DSM-III) (American Psychiatric Association, 1980) distinguished an adjustment disorder as meeting the following criteria:

A. A maladaptive reaction to an identifiable psychological stressor, that occurs within three months of the onset of the stressor.

$B$. The maladaptive nature of the reaction is indicated by either of the following: (1) impairment in social or occupational functioning (2) symptoms that are in excess of a normal and expectable reaction to the stressor.

C. The disturbance is not merely one instance of a pattern of overreaction to stress. ... 
D. It is assumed that the disturbance will eventually remit after the stressor ceases or ... when a new level of adaptation is achieved. (pp. 300-301)

A final caveat noted that the disturbance should not meet the criteria for any other more appropriate diagnostic category.

Whether one views the response of the NDEr as normal or maladaptive in quality and degree, it is apparent that a process of adjustment of generally significant proportions must be undertaken. Regardless of the nature and extent of the adaptation to be achieved, the therapeutic goal should be to ease and enhance reorientation to the demands of the environment, given the frame of reference of the experience.

\section{Cross-Cultural Counseling and World Views}

The common denominator of the research literature on NDEs is its recurring reports of a basic change in the world view of experiencers. As a result of having undergone that process, as outlined above, the individual comes to identify with a frame of reference that is significantly different from that of the predominant culture. Viewed in that fashion, the NDEr is a member of a cultural minority, whose beliefs, values, and attitudes conflict with those of the majority culture. (Recall that most studies have relied on samples taken from the adult American population; the majority culture referred to, then, is that of the Western world and its Judeo-Christian heritage. Very different findings, in terms of aftereffects and adjustment, may emerge from non-Western cultures.)

Appropriate intervention strategies, therefore, would best be generated from a cross-cultural paradigm. Very often the term "cross-cultural" is wrongfully equated with geography or ethnic group; while those are components of the paradigm, here it is used in its broader connotation as an area concerned with investigating differences in world views and in developing intervention strategies that take into account the value system of the client.

Despite the unusual circumstances through which the reorientation occurs, the process NDErs undergo is not unique to that, or any other, specific group attempting cultural adaptation. The difficulties reported by NDErs bear a striking resemblance to those associated with culture shock, i.e., anxiety, depression, difficulty in discerning appropriate responses, and barriers to communication. If one considers the world view experienced during the process of the NDE as the "home culture," and 
the world view of this realm as the "host culture," then Paul Pedersen's (1981) description could easily apply to the NDEr:

The specific symptoms of culture shock focus on either dissatisfaction with the host culture or idealization of the home culture. The host culture is criticized as being peculiar, irrational, inefficient, and unfriendly. The visitor is likely to fear being taken advantage of, being laughed at or talked about, not being accepted, and wanting to spend more time around persons from the visitor's home culture. ... Minor annoyances in the host culture become exaggerated, and the few remaining links with the home culture become extraordinarily important. ... Culture shock is likely to extend over a long period of time, reappear in a variety of forms, and not be limited to an initial adjustment. ... The visitor experiences a genuine identity crisis, requiring either that the former identity be disowned or that the visitor create and maintain multiple identities. . . . In either case, the visitor is required to reintegrate, confront, and challenge the basic underlying assumptions of his or her personality. (p. 319)

While the impetus for change is, for NDErs, group-specific, the problems are not.

Using a cross-cultural paradigm, it is clear that the process of adjustment following the NDE is a normal response, demonstrably similar to that of culture shock, and, as such, tends to follow a predictable pattern (Adler, 1975). The first stage involves the initial contact with what above was referred to as the host culture; for the NDEr that is the period immediately following the awareness of returning to the physical body. According to Peter Adler, the stage "is marked by the excitement and euphoria of the new experience," and in which "the individual is still functionally integrated with his or her own culture" (p. 16), i.e., that of the NDE. The second stage is characterized by confusion and disorientation, as the individual becomes increasingly aware that his or her attitudes and values conflict with those of the majority culture. Inappropriate behaviors and interpersonal misunderstandings contribute to one's sense of being different, and of feeling isolated. Alienation, depression, and withdrawal are common reactions. During the third stage, the individual may actively cultivate relationships with only those who share his or her culture, i.e., other NDErs. A strong rejection of the host culture is likely. However, "rejection of the culture that causes negative feelings is more than a reactive behavior and becomes the basis for new, intuitive, emotional, and cognitive experiences" (p. 17). The fourth stage involves a gradual increase in coping skills and personal flexibility, along with a degree of comfort in one's status as both insider and outsider. The fifth, and final, transitional stage is marked by an acceptance 
of cultural similarities and differences. The individual is able to give, as well as to inspire, a demeanor of sensitivity and trust. Expressiveness, humor, and creativity re-emerge in a heightened fashion, in appreciation of difference and diversity. Although the stages are listed sequentially, the process is not a linear one. The client may be functionally in more than one stage, with "progression" in some areas and "regression" in others occurring simultaneously.

While the adjustment process tends to follow this pattern, there is considerable variation from individual to individual in the quality and degree to which the transitional behaviors are manifested. Personality, coping skills, and environmental variables, among other factors, can all contribute to the ease or dis-ease with which the transition is negotiated. One important factor seems to be the magnitude of the difference between the home and host cultures. It may be, then, that NDErs who have had a particularly deep or prolonged experience would have greater difficulty adjusting.

Given this generic frame of reference from which to view the adjustment problems of NDErs, the issue then becomes how best to facilitate the adjustment process; in the language of the psychologist, that means developing a treatment plan. As noted earlier, interventions designed for the NDEr are more appropriately drawn from nonmedical and nonpsychoanalytic traditions, given that the NDE is not a pathological process. In actuality, then, the practitioner is not so much developing a "treatment" plan, as he or she is establishing a working model through which to enlist the aid of the client in determining how best to negotiate this stage of development. This shift in semantics and cognition is not a trivial one, if the practitioner is genuinely to consider the NDEr a client, and not a patient. Conceptually, then, the paradigm is crosscultural, and the exemplar, the psychoeducational model; specific intervention strategies must flow from both.

\section{Therapist Variables}

Too often a consideration of therapist variables is neglected, even though it is, implicitly, the first step in formulating an intervention strategy. When employing a cross-cultural framework, at least three issues must be considered: 1 . the world view of the therapist, 2 . the world view of the client, and 3 . the world view of the therapist vis-à-vis the world view of the client. Prior to engaging in any form of therapy, it is important that the therapist be overtly aware of his or her value system. In the cross-cultural encounter, that is an imperative (Ibrahim, 
1985). Working with someone of a significantly different culture is "likely to highlight otherwise hidden conflicts in our own behavior at a rate many times faster than when working within our own familiar culture" (Pedersen, 1981, p. 321). For the benefit of the client, these issues must be resolved to the greatest extent possible prior to entering into the therapeutic relationship. In adopting a cross-cultural paradigm, the practitioner is ethically bound to be knowledgeable of the client's cultural reference group, and to relate with sensitivity (Sundberg, 1981). Finally, as a rule, it is considered best that the therapist and client in a cross-cultural encounter share the same world view (Sue, 1981).

The practitioner who chooses to work with NDErs may be particularly susceptible to conflict, both internally and in the dyadic relationship (Bush, personal communication, November 5, 1984). This involves not only the inclination on the part of many to equate the NDE with some form of medical or psychological disturbance, but it also includes a tendency on the part of others to beatify it. A professional stance necessitates the often delicate balancing of empathic involvement with objective distance. It seems likely that given the attitude set of a "typical" NDEr that successfully treading that fine line is a precarious process.

One might reasonably argue, therefore, that the best therapist for the NDEr is another NDEr. Hopefully that is not necessarily the case. Not only may he or she be too involved in a personal struggle to adjust to be of help, but that might also reinforce the existing tendency to associate primarily or exclusively with other NDErs and thus impede the adjustment process.

What is proposed here is that the therapist 1 . be thoroughly acquainted with the NDE and its aftereffects, 2 . assume a cross-cultural perspective, and 3 . be sympathetic to non-Western philosophies, particularly those of the Far East, and to mystical traditions.

\section{Assessment Techniques}

Once therapist variables are accounted for, the issue then becomes how to assess the client, that is, how to explore the nature and quality of the NDE, and how to determine to what extent the client's experience conforms to group norms. In addition to providing the therapist with necessary "diagnostic" information, assessment techniques should also be tied to the desired outcome of the therapy program (Gottman \& Markman, 1978). The information gathered early in the therapeutic relationship should assess the issues, as well as provide a baseline from which to evaluate the success of the intervention. 
Depending upon when during the post-NDE period the practitioner becomes involved, some assessment techniques may be more appropriate than others. Reconsideration of the adjustment stages and their accompanying behaviors suggests that during the earliest stages of adjustment the more passive, empathic listening modes would least alienate the client, combined perhaps with basic forms of reality testing, i.e., orienting the client to time, place, and person. During later stages, basic interviewing techniques that employ empathic, facilitative styles should suffice.

These techniques should be familiar to experienced therapists, particularly those trained in counseling psychology and related disciplines. What is needed is for the therapist to use the initial contacts to 1. discern the nature of the client's experience (NDE vs. non-NDE), 2. ascertain the stage of adjustment the client may be in, 3 . enlist from the client what type of help might be useful, and 4. establish a baseline, via interview and behavioral observation techniques, from which to evaluate change.

\section{Intervention Strategies}

A major premise of this paper has been that most practitioners already possess the requisite skills for aiding this particular population. What has been needed is a framework from which to proceed. If the interventionist accepts the cross-cultural perspective, and is willing to employ the psychoeducational model, then virtually any of the commonly used therapeutic techniques should also be effective for the NDEr. This would include, but not be limited to, individual, group, and family therapy and crisis intervention. Thus, the specifics of what strategy to use and when would be best left to the discretion and expertise of the therapist.

Often neglected, however, is a consideration of the uniqueness of the individual, apart from the group with which he or she has been identified. As has been stressed throughout, there may be considerable individual variation in the nature and quality of the NDE, and in the process of adjustment. The latter depends, in part, on the degree of difference between the pre- and post-NDE world views, and on the degree of difference between the post-NDE world view of the experiencer, and that of the culture and significant others.

In order to individualize the intervention strategy to the needs of the NDEr and significant others, it is proposed that the Scale to Assess World Views Across Cultures (SAWVAC) (Tbrahim \& Kahn, 1984) be considered as an educational tool for use during the therapeutic process. 
The scale is grounded in existential theory, and uses "a set of universal constructs of value emphases and value orientations to understand our own and others' world views. Specifically, it examines one's views of human nature, human relationships, nature, time, and activity. As Ibrahim (1984) noted:

Starting with an analysis of the client's world view as the first source of information, we are freed from perpetuating stereotypes and biases that exist regarding the client's specific group.

The client-specific information gained through SAWVAC can be placed in the context of the client's primary group information and in the larger context of the majority culture. ... i.e., is the individual alienated from him or herself, alienated from own primary culture or alienated from the larger majority culture. (pp. 8-9)

In the absence of group data for NDErs, using this scale, it can nonetheless prove extremely useful in highlighting pre- and post-NDE value changes, and for examining differences between the world view of the NDEr and the world views of relevant others. (Should a data base using the SAWVAC accumulate, the scale could then be better considered as a part of the assessment process, as well as a potential research tool.)

\section{Benefits and Limitations}

The primary strength of the position of this paper is the functional integration of two heretofore distinct areas of research and practice. The conceptual linking of cross-cultural counseling with the near-death experience should provide a broad range of practitioners with the basis from which to successfully intervene in the adjustment process of NDErs. However, until the model is field tested and critically researched, it remains at the level of theoretical speculation.

\section{References}

Adler, P. (1975). The translation experience: An alttenative view of culture shock. Journal of Humanistic Psychology, 15(4), 13-23.

American Psychiatric Association. (1980). Diagnostic and Statistical Manual of Mental Disorders (3rd ed.). Washington, DC: American Psychiatric Association.

American Psychological Association Committee on Standards for Providers of Psychological Services. (1981). Specialty guidelines for the delivery of services by counseling psychologists. American Psychologist, 36, 652-663.

Atwater, P. M. H. (1983). Coming back: The aftereffects of survival. Vital Signs, 3(2), 16. Eysenck, H. J. (Ed.). (1972). Encyclopedia of Psychology. New York, NY: Herder \& Herder. 
Gallup, G., Jr. (1982). Adventures in Immortality: A Look Beyond the Threshold of Death. New York, NY: McGraw-Hill.

Gottman, J., \& Markman, H. (1978). Experimental designs in psychotherapy research. In S. Garfield \& A. Bergin (Eds.), Handbook of Psychotherapy and Behavior Change (2nd ed.). New York, NY: John Wiley \& Sons.

Goldenson, R. M. (1984). Longman Dictionary of Psychology and Psychiatry. New York, NY: Longman, Inc.

Greyson, B. (1982). Near-death studies, 1981-1982: A review. Anabiosis, 2, 150-158.

Greyson, B., \& Flynn, C. (Eds.). (1984). The Near-Death Experience: Problems, Prospects, Perspectives. Springfield, IL: Charles C. Thomas.

Ibrahim, F. (1985). Effective cross-culturally counseling and psychotherapy: A framework. Counseling Psychologist, 13, 625-638.

Ibrahim, F. (1984). Cross-cultural counseling and psychotherapy: Initial client assessment. Unpublished manuscript, University of Connecticut, Storrs.

Ibrahim, F., \& Kahn, H. (1984). Scale to Assess World Views Across Cultures (SAWVAC). Unpublished manuscript, University of Connecticut, Storrs.

Moody, R., Jr. (1975). Life After Life. Covington, GA: Mockingbird Books.

Moody, R., Jr. (1977). Reflections on Life After Life New York, NY: Bantam.

Pedersen, P. (1981). Alternative futures for cross-cultural counseling and psychotherapy. In A. J. Marsella \& P. B. Pederson (Eds.), Cross-Cultural Counseling and Psychotherapy (2nd ed.). New York, NY: Pergamon Press.

Ring, K. (1980). Life at Death. New York, NY: Coward, McCann \& Geoghegan.

Ring, K. (1984). Heading Toward Omega New York, NY: William Morrow.

Sabom, M. (1981). Recollections of Death: A Medical Investigation. New York, NY: Harper \& Row.

Sue, D. (1981). Counseling the Culturally Different. New York, NY: John Wiley \& Sons.

Sundberg, N. (1981). Cross-cultural counseling and psychotherapy: A research overview. In A. J. Marsella \& P. B. Pedersen (Eds.), Cross-Cultural Counseling and Psychotherapy (2nd ed.). New York, NY: Pergamon Press.

Whiteley, J. (Ed.). (1984). Counseling psychology: A historical perspective. Counseling Psychologist, 12, 1.

Wolman, B. B. (Ed.). (1973). Dictionary of Behavioral Science. New York, NY: Van Nostrand Reinhold. 